\title{
JADD Announcements for June 2010
}

Yale Child Study Center Autism Program

9th Annual Summer Institute on Autism Spectrum Disorders

Monday-Friday

July 12-16, 2010

Yale School of Medicine, Harkness Auditorium 333 Cedar Street

New Haven, CT

The Yale School of Medicine and Yale Child Study Center have scheduled this conference to be held on July 12-16, 2010 at The Yale School of Medicine Harkness Auditorium. The institute will include over 20 faculty members from the Yale Child Study Center, as well as the Center for Children with Special Needs.

Autism Spectrum Disorders (ASDs) are characterized by early onset socialization and communication impairments, as well as patterns of restricted interests and behavioral rigidities, that affect individuals across their lifespan. The number of individuals with ASD obtaining cognitive levels at or above the normative range is increasing. Yet, many of these individuals still fail to translate their potential into adaptive, real-life skills.

This stresses the need for education of professionals, pediatricians, psychiatrists, geneticists, general practitioners, nurses, educators, speech pathologists, psychologists, social workers, occupational therapists, physical therapists, and any professional in contact with children in a medical, clinical, or educational setting.

Topics will include:

- Autism Spectrum Disorders: Social Cognition

- Clinical Assessment \& Evaluation of ASD

- Behavioral Assessment and Intervention

- Autism in Infancy \& Early Childhood

- Treatment \& Educational Programming

This educational activity is designated for a maximum of 28 AMA PRA Category 1 Credits $^{\text {TM }}$

To register and view the conference program, please visit YaleCME: www.cme.yale.edu (click on 'CME Conference Schedule \& Registration') or call 203-785-4578 